\title{
(6) OPEN ACCESS \\ Imaging diagnoses and outcome in patients presenting for primary angioplasty but no obstructive coronary artery disease
}

\author{
Tarun K Mittal, ${ }^{1}$ Luise Reichmuth, ${ }^{2}$ Ben Ariff, ${ }^{3}$ Praveen P G Rao, ${ }^{4}$ Aigul Baltabaeva, ${ }^{1}$ \\ Shelley Rahman-Haley, ${ }^{1}$ Tito Kabir, ${ }^{1}$ Joyce Wong, ${ }^{1}$ Miles Dalby ${ }^{1}$
}

'Department of Cardiology \& Cardiac Imaging, Harefield Hospital, Royal Brompton \& Harefield NHS Foundation

Trust, London, UK

${ }^{2}$ Department of Imaging,

Mater Dei University Hospital, Malta, Malta

${ }^{3}$ Department of Imaging, Imperial College NHS Healthcare Trust, London, UK ${ }^{4}$ Department of Imaging, Glenfield Hospital, University Hospital of Leicester, Leicester, UK

\section{Correspondence to}

Dr Tarun K Mittal, Department of Cardiac Imaging, Harefield Hospital, Royal Brompton \& Harefield NHS Foundation Trust, Hill End Road, Middlesex, London UB9 6JH UK; t.mittal@imperial.ac.uk

Received 18 November 2015 Revised 18 April 2016 Accepted 16 May 2016 Published Online First 1 July 2016
CrossMark

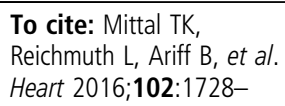

ABSTRACT

Objective A proportion of patients with suspected ST-elevation myocardial infarction (STEMI) presenting for primary percutaneous coronary intervention $(\mathrm{PPCl})$ do not have obstructive coronary disease and other conditions may be responsible for their symptoms and ECG changes. In this study, we set out to determine the prevalence and aetiology of alternative diagnoses in a large PPCI cohort as determined with multimodality imaging and their outcome.

Methods From 2009 to 2012, 5238 patients with suspected STEMI were referred for consideration of $\mathrm{PPCl}$. Patients who underwent angiography but had no culprit artery for revascularisation and no previous history of coronary artery disease were included in the study. Troponin values, imaging findings and all-cause mortality were obtained from hospital and national databases. Results A total of $575(13.0 \%)$ patients with a mean age of $58 \pm 15$ years ( $69 \%$ men) fulfilled the inclusion criteria. A specific diagnosis based on imaging was made in 237 patients $(41.2 \%)$ including cardiomyopathies $(n=104,18 \%)$, myopericarditis $(n=48$, $8.4 \%$ ), myocardial infarction/other coronary abnormality $(n=27,4.9 \%)$ and severe valve disease $(n=23,4 \%)$. Pulmonary embolism and type $\mathrm{A}$ aortic dissection were identified in seven (1.2\%) and four $(0.7 \%)$ cases respectively. A total of $40(7.0 \%)$ patients died over a mean follow-up of 42.6 months.

Conclusions A variety of cardiac and non-cardiac conditions are prevalent in patients presenting with suspected STEMI but culprit-free angiogram, some of which may have adverse outcomes. Further imaging of such patients could thus be useful to help in appropriate management and follow-up.

\section{INTRODUCTION}

The diagnosis of ST-elevation myocardial infarction (STEMI) is made from a history of acute-onset chest pain, ST-elevation on ECG and subsequent release of cardiac biomarkers suggesting myocardial necrosis. ${ }^{1}$ Guidelines recommend urgent revascularisation, ideally with primary percutaneous coronary intervention (PPCI) of the culprit artery. ${ }^{1}$ The levels of biomarkers such as troponin are generally not available at the time of revascularisation.

A subset of patients (2.6\%-14\%) with suspected STEMI do not have a culprit lesion or obstructive coronary artery disease (CAD) on the initial diagnostic coronary angiogram (DCA). ${ }^{2-5}$ Some of these patients may have other non-atheromatous cardiac or non-cardiac causes for their symptoms and ECG abnormalities as well as biomarker release. $^{67}$ In the last few years, there has been an increasing interest in alternative causes of acute chest pain associated with troponin rise particularly with non-invasive diagnosis of myocarditis on cardiac magnetic resonance (CMR) or demonstration of myocardial infarction (MI) in the presence of recanalised arteries. ${ }^{8-10}$ However, such studies have mainly focused on small series of patients presenting within a broader spectrum of acute coronary syndrome (ACS) with raised troponin with the aim of evaluating the usefulness of CMR. The prevalence of non-coronary causes of acute chest pain and their prognosis, particularly those presenting with suspected STEMI, is less well described.

The aim of this study was to determine the prevalence and outcome of different cardiac and non-cardiac conditions as identified on multimodality imaging in a large cohort of patients presenting with suspected STEMI, who had no culprit lesion on the angiogram.

\section{METHODS}

\section{Patient recruitment}

Our institution is a tertiary cardiothoracic centre with an established 24-hour PPCI service with a catchment population of about 2 million people. Patients with suspected STEMI on the basis of clinical signs and symptoms, and 12-lead ECG are transferred directly by the ambulance service (diagnosis made autonomously in the field without the use of teletransmission) to our centre for consideration of PPCI. ${ }^{11}$ Patients on arrival are reviewed by the on-call cardiology team on site to confirm the diagnosis and proceed to PPCI, if appropriate. Some patients are also transferred from nearby general hospitals, if they are found to have suffered a STEMI.

Consecutive patients who were admitted to the PPCI programme between 1 January 2009 and 31 December 2012 were identified from the programme registry $(n=5238)$. Patients were excluded as per the criteria shown in figure 1. All patients without a culprit lesion on angiogram and no known CAD were included in the study. Absence of a culprit lesion was defined as absence of a significant stenosis ( $>70 \%$ luminal narrowing) in the vessel supplying the area of acute ECG change. Known CAD was defined as history of previous ACS, PCI or coronary artery bypass surgery. 


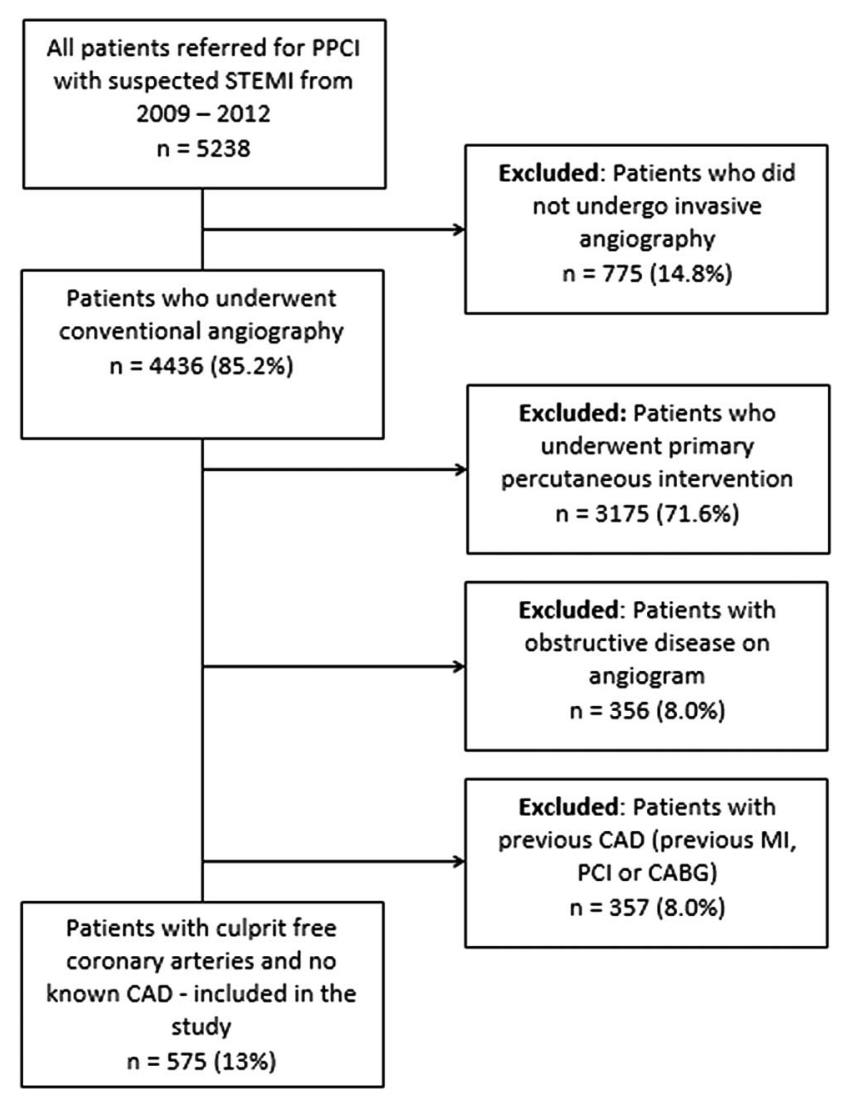

Figure 1 Flow chart showing exclusion criteria and final patient population included in the study. CABG, coronary artery bypass surgery; CAD, coronary artery disease; $\mathrm{MI}$, myocardial infarction; $\mathrm{PCl}$, percutaneous coronary intervention; $\mathrm{PPCl}$, primary percutaneous coronary intervention; STEMI, ST-elevation myocardial infarction.

The National Health Service ethics approval was obtained from the hospital's research department (Project ID: 199060).

\section{Data collection and imaging indications}

Patient demographics, traditional risk factors for CAD and presenting ECG as recorded in the database were identified. For all included patients, the highest initial troponin result during the admission was recorded (Access AccuTnI assay, Beckman Coulter, Brea, CA, USA) from laboratory results in the electronic patient records (EPR). The different non-invasive imaging procedures performed as a result of the primary admission episode, their findings and subsequent management were recorded from the hospital Picture Archiving and Communication System and the EPR. Images were not reviewed in all cases unless there was uncertainty about a diagnosis or clarification was required. Our hospital has a protocol for routine performance of plain chest X-rays (CXR) and echocardiograms for all PPCI admissions once the patient is stable. CMR is performed in those with non-obstructed coronary arteries on DCA and unexplained rise in troponin or if required from echocardiogram findings. A chest or ECG-gated cardiac CT is performed if clinically indicated in patients with suspected pulmonary embolism (PE), acute aortic dissection, pericarditis or other coronary abnormality not identified on initial DCA. All imaging was performed within the acute admission with follow-up scans as required.

\section{Imaging equipment and protocol}

Echocardiography was performed on different commercially available ultrasound machines (Vivid-7 or 9, GE Healthcare,
Milwaukee, WI, USA and IE33, Phillips Healthcare, Andover, MA, USA). CT scans of the chest or ECG-gated scans of the heart were carried out on a 64-slice scanner (Aquilion 64, Toshiba Medical Systems, Japan). CMR scans of the heart were performed on a cardiac optimised 1.5T system (Avanto, Siemens Medical Systems, Germany). Standard protocols were used for all imaging as recommended and described by various societies, ${ }^{12-14}$ with echocardiograms being performed by British Society of Echocardiography certified sonographers and cardiologists. CT and CMR interpretation was carried out by level 3 certified cardiothoracic radiologists (TKM, BA) and/or imaging cardiologists (JW, SR-H).

\section{Imaging diagnostic criteria and outcome}

All imaging diagnoses (called primary diagnosis) were made according to standard criteria using one or more imaging modalities and classified under a broader diagnostic category. In some patients, additional imaging findings were recorded if they were considered important and not being a result of the primary diagnosis.

Myocarditis was diagnosed on CMR as per the Lake Louise consensus criteria based on T2-weighted oedema and late gadolinium enhancement (LGE) sequences. ${ }^{15}$ A diagnosis of pericarditis was made in the presence of a pericardial effusion or thickening on any imaging modality in the absence of other findings. MI was diagnosed if there was a typical LGE pattern on CMR involving the subendocardium. ${ }^{16}$ Dilated cardiomyopathy (DCM) was defined as left ventricular (LV) dilatation with generalised hypokinesia and ejection fraction (EF) of $<50 \%$ in the absence of CAD or valvular disease. ${ }^{17}$ Hypertrophic cardiomyopathy (HCM) was diagnosed in the presence of unexplained myocardial thickness of $\geq 15 \mathrm{~mm}$ on echocardiography and CMR in the absence of another cardiac or systemic disorder that could explain the hypertrophy. ${ }^{18}$ A diagnosis of Takotsubo cardiomyopathy (TTCM) was made when there was dilatation and dysfunction of the distal half of the LV on ventriculogram, which normalised on subsequent imaging scan in the absence of MI or other significant pathologies. ${ }^{19}$ Hypertensive heart

Table 1 Patient characteristics and imaging performed

\begin{tabular}{|c|c|c|}
\hline & Number $(n=575)$ & Percentage \\
\hline Age, mean $( \pm S D)$ & $57.7(15.2)$ & \\
\hline Sex, male (\%) & 397 & 69 \\
\hline Hypertension & 196 & 34.1 \\
\hline Dyslipidaemia & 139 & 24.2 \\
\hline Current smoking & 114 & 19.8 \\
\hline Diabetes & 74 & 12.9 \\
\hline Family history of premature CAD & 112 & 19.5 \\
\hline Out-of-hospital cardiac arrest & 14 & 2.4 \\
\hline ST-elevation/LBBB on ECG & 381 & 66.3 \\
\hline Troponin raised & 229 & 39.8 \\
\hline Troponin, median (IQR) & $0.48(0.11,4.0)$ & \\
\hline Chest X-ray & 370 & 64.4 \\
\hline Echocardiogram & 422 & 73.4 \\
\hline CT scan & 100 & 17.4 \\
\hline CMR scan & 147 & 25.6 \\
\hline $\mathrm{EF}<50 \%$ * & 75 & 13.0 \\
\hline CMR LGE & 72 & 12.5 \\
\hline
\end{tabular}


disease was defined as concentric myocardial thickening $>12 \mathrm{~mm}$ associated with systolic and/or diastolic dysfunction in patients with a known long-standing history of hypertension or newly diagnosed persistent hypertension for the purpose of this study. $^{20}$ Different valvular diseases were included if they were found to be severe in degree as per guidelines. ${ }^{21}$ Pulmonary infection was defined as air space shadowing on the CXR or CT, associated with raised inflammatory markers. Filling defects in the pulmonary arteries on CT pulmonary angiogram were diagnostic of PE. Aortic pathology included patients with imaging findings of acute aortic dissection (type A or B), intramural haematoma or unknown dilatation of the aortic root and/ or thoracic aorta of $\geq 5 \mathrm{~cm}$ in maximum diameter.

Finally, patient outcome was determined as all-cause mortality from UK's Health \& Social Care Information Centre, which tracks deaths of people residing within the country and registered with a general practitioner. Cause of death was identified from hospital records and general practitioners.

\section{Statistical methods}

Continuous variables that were normally distributed are expressed as mean $\pm S D$. Other continuous variables such as troponin levels that were not normally distributed are described as median with interquartile range and their groups were analysed using Mann-Whitney test. Categorical variables are expressed as percentages and the $\chi^{2}$ test was performed for analysis. A two-tailed $\mathrm{p}$ value of $<0.05$ was considered statistically significant.

Cox regression with univariable and multivariable analyses was performed to examine factors associated with survival

Table 2 Primary imaging diagnosis

\begin{tabular}{|c|c|c|c|c|c|c|c|}
\hline & Number $(n=575)$ & Per cent* & Per cent $t$ & Age, $\operatorname{mean}_{ \pm} \mathrm{SD}$ & $\%$ Maleł & Raised troponin $¥(\%)$ & $\begin{array}{l}\text { Died } \neq \\
\mathrm{n}=40(\%)\end{array}$ \\
\hline \multicolumn{8}{|l|}{ Imaging diagnosis } \\
\hline No imaging & 76 & 13.2 & NA & $57 \pm 15$ & 72 & 0 & 0 \\
\hline Imaging but no diagnosis & 262 & 45.6 & NA & $57 \pm 15$ & 77 & $58(22)$ & $13(5)$ \\
\hline Imaging with diagnosis & 237 & 41.2 & NA & $59 \pm 15$ & 59 & $171(72)$ & $27(11)$ \\
\hline All cardiomyopathy & 104 & 18.1 & 43.9 & $60 \pm 14$ & 52 & $71(68)$ & $9(9)$ \\
\hline Hypertrophic cardiomyopathy & 28 & 4.9 & 11.8 & $55 \pm 11$ & 82 & $15(54)$ & $2(7)$ \\
\hline Dilated cardiomyopathy & 18 & 3.1 & 7.6 & $54 \pm 16$ & 67 & $13(72)$ & $2(11)$ \\
\hline Takotsubo cardiomyopathy & 27 & 4.7 & 11.4 & $70 \pm 10$ & 4 & $23(85)$ & $3(11)$ \\
\hline Hypertensive cardiomyopathy & 10 & 1.7 & 4.2 & $61 \pm 14$ & 70 & $7(70)$ & 0 \\
\hline LVD with tachyarrhythmia or bradyarrhythmia & 6 & 1.0 & 2.5 & $61 \pm 14$ & 50 & $5(83)$ & $1(17)$ \\
\hline LVD with LBBB & 4 & 0.7 & 1.7 & $51 \pm 17$ & 50 & 0 & 0 \\
\hline LVD of non-specific aetiology & 10 & 1.7 & 4.2 & $58 \pm 13$ & 60 & $7(70)$ & $1(10)$ \\
\hline Myopericarditis & 48 & 8.4 & 20.3 & $51 \pm 17$ & 71 & $40(83)$ & $2(4)$ \\
\hline Myocarditis & 36 & 6.3 & 15.2 & $47 \pm 16$ & 69 & $35(97)$ & $1(3)$ \\
\hline Pericarditis & 13 & 2.3 & 5.5 & $63 \pm 14$ & 69 & $6(46)$ & $1(8)$ \\
\hline Ml/coronary abnormality & 27 & 4.9 & 11.4 & $53 \pm 11$ & 71 & $27(93)$ & $1(4)$ \\
\hline MI & 21 & 3.6 & 8.9 & $53 \pm 11$ & 76 & $21(100)$ & $1(5)$ \\
\hline Anomalous coronaries & 2 & 0.3 & 0.8 & $58 \pm 7$ & 100 & $1(50)$ & 0 \\
\hline Coronary spasm & 3 & 0.5 & 1.3 & $56 \pm 16$ & 33 & $2(67)$ & 0 \\
\hline Coronary artery dissection & 1 & 0.2 & 0.4 & $46 \pm 0$ & 0 & $1(100)$ & 0 \\
\hline Valve disease, severe & 23 & 4.0 & 9.7 & $71 \pm 13$ & 43 & $17(74)$ & $8(35)$ \\
\hline Aortic valve stenosis & 16 & 2.8 & 6.8 & $72 \pm 14$ & 38 & $12(75)$ & $5(31)$ \\
\hline Aortic valve regurgitation & 4 & 0.7 & 1.7 & $71 \pm 11$ & 75 & $3(75)$ & $2(50)$ \\
\hline Mitral valve disease & 3 & 0.5 & 1.3 & $66 \pm 19$ & 33 & $2(67)$ & $1(50)$ \\
\hline Cardiac other & 3 & 0.5 & 1.3 & $73 \pm 21$ & 33 & $2(67)$ & $1(33)$ \\
\hline Pulmonary hypertension & 2 & 0.3 & 0.8 & $86 \pm 5$ & 0 & $1(50)$ & $1(50)$ \\
\hline PFO & 1 & 0.2 & 0.4 & $48 \pm 0$ & 100 & $1(100)$ & 0 \\
\hline Aortic abnormality & 6 & 1.0 & 2.5 & $54 \pm 12$ & 68 & $2(33)$ & $2(33)$ \\
\hline Aortic dissection & 4 & 0.7 & 1.7 & $56 \pm 15$ & 75 & $2(50)$ & $2(50)$ \\
\hline Aortic aneurysm & $2(3 \S)$ & 0.3 & 0.8 & $50 \pm 4$ & 50 & 0 & 0 \\
\hline Pulmonary abnormality & 23 & 4.0 & 9.7 & $64 \pm 14$ & 68 & $14(56)$ & $3(13)$ \\
\hline Infection & $12(5 \S)$ & 2.1 & 5.1 & $67 \pm 12$ & 92 & $5(42)$ & $2(17)$ \\
\hline Pulmonary embolism & 7 & 1.2 & 3.0 & $58 \pm 14$ & 43 & $6(86)$ & 0 \\
\hline Pulmonary neoplasia & $3(2 \S)$ & 0.5 & 1.3 & $67 \pm 3$ & 67 & $2(67)$ & $1(33)$ \\
\hline Pneumothorax & 1 & 0.2 & 0.4 & $35 \pm 0$ & 100 & 0 & 0 \\
\hline Abdominal abnormality & 3 & 0.5 & 1.3 & $72 \pm 17$ & 33 & $1(33)$ & $1(33)$ \\
\hline Cholecystitis & 2 & 0.3 & 0.8 & $70 \pm 23$ & 50 & $1(50)$ & 0 \\
\hline Pancreatitis & 1 & 0.2 & 0.4 & $77 \pm 0$ & 0 & 0 & $1(100)$ \\
\hline
\end{tabular}


times. All variables were entered into the multivariable analysis regardless of statistical significance. Data were collected from all patients for all variables except for EF, which was only available for 429 patients from echocardiography and CMR. Kaplan-Meier survival graphs were drawn with survival times measured from the time of acute presentation to the time of death. Patients who did not die were censored at the time of last follow-up. Assumptions of proportional hazards were checked by graphing $-\operatorname{In}\{-\operatorname{In}($ survival) $\}$ against $\operatorname{In}$ (analysis time).

All statistical analysis was performed using Stata V.13.1 (StataCorp LP, College Station, TX, USA).

\section{RESULTS}

During the 4-year study period, 575 patients (13.0\%) fulfilled the inclusion criteria. The mean age of the included patients was 57.7 years $( \pm 15.2)$ with $397(69 \%)$ being men. Two hundred twenty-nine patients $(39.8 \%)$ had a raised troponin. Other baseline characteristics are given in table 1 .

A total of 499 patients $(86.8 \%)$ had one or more imaging procedure performed with a specific imaging-based diagnosis made in 237 patients (41.2\%) (table 2). Besides, there were 10 additional important findings including 3 cases of aortic aneurysm, 5 cases of pulmonary infection and 2 cases of additional pulmonary cancers that were present apart from the primary diagnosis (table 2).

The different imaging modalities used for the various diagnostic categories are given in table 3. Multiple imaging techniques were used in most cases as thought to be appropriate to arrive at a diagnosis.

Out of the patients who had one or more imaging test performed, 229 (46\%) were troponin positive (TP). Among these, an imaging diagnosis was made in 171 patients (74.7\%). One hundred twenty-two (53\%) TP patients underwent CMR scan as the rest either had diagnosis made from other imaging techniques or were considered unsuitable (due to low clinical risk or contraindications). The TP patients who did not undergo a CMR scan and had no diagnosis made on other imaging modalities had a much lower troponin level (median $0.095 \pm 0.48$ vs $1.58 \pm 11.55$ in those with diagnosis, $\mathrm{p}<0.0001$ ). Of the 270 (54\%) troponin-negative patients, an imaging diagnosis was made in 66 patients $(24.4 \%)$.

A total of 40 deaths $(7.0 \%)$ from all causes occurred during a mean follow-up of $42.6 \pm 17$ months with mortality varying widely between different conditions (table 2). Twelve of these deaths occurred within 30 days giving a 30-day all-cause mortality rate of $2.1 \%$. Different causes of death and mean time to death is given in table 4. Cox regression revealed that on multivariate analysis, only age, out-of-hospital cardiac arrest (OHCA) and $\mathrm{EF}<50 \%$ were significantly associated with survival times (table 5). None of the patients with LGE on CMR died. Kaplan-Meier survival curves demonstrating the effect of significant variables on survival are given in figure 2 .

\section{DISCUSSION}

Our study demonstrates the prevalence of alternative diagnoses in patients admitted with suspected STEMI who had culprit-free coronary arteries on angiography and no known CAD. This is the largest study of patients presenting to a large PPCI programme with further clinically directed but comprehensive multimodality non-invasive imaging. It demonstrates that just under half the patients are discharged with an alternative diagnosis, some of which are potentially associated with an adverse outcome. However, not all the diagnoses may be responsible for patients' presentation and some could be purely coincidental.
Table 3 Imaging modalities used for different diagnostic categories

\begin{tabular}{|c|c|c|c|c|c|}
\hline $\begin{array}{l}\text { Imaging } \\
\text { diagnosis }\end{array}$ & $\begin{array}{l}\text { Number } \\
(\mathrm{n}=499)\end{array}$ & CXR (\%)* & $\mathrm{ECHO}(\%)^{*}$ & CT (\%)* & CMR $(\%)^{*}$ \\
\hline $\begin{array}{l}\text { Imaging but no } \\
\text { diagnosis }\end{array}$ & 262 & $200(76)$ & $196(75)$ & $38(15)$ & $28(11)$ \\
\hline Cardiomyopathy & 104 & $71(68)$ & $100(96)$ & $15(14)$ & $55(53)$ \\
\hline Myopericarditis & 48 & $34(71)$ & 47 (98) & $12(25)$ & $37(77)$ \\
\hline $\begin{array}{l}\text { Ml/coronary } \\
\text { abnormality }\end{array}$ & 27 & $15(56)$ & $27(100)$ & $6(22)$ & $20(74)$ \\
\hline Valve disease & 23 & $19(83)$ & $23(100)$ & $6(26)$ & $2(9)$ \\
\hline $\begin{array}{l}\text { Pulmonary } \\
\text { abnormality }\end{array}$ & 23 & $22(96)$ & $18(78)$ & $15(65)$ & $2(9)$ \\
\hline $\begin{array}{l}\text { Aortic } \\
\text { abnormality }\end{array}$ & 6 & $4(67)$ & $5(83)$ & $3(50)$ & $2(33)$ \\
\hline Others & 6 & $5(83)$ & $6(100)$ & $5(83)$ & $1(17)$ \\
\hline
\end{tabular}

${ }^{*}$ All percentages are from the diagnostic category in the row itself. More than one modality was used for diagnosis where appropriate.

CMR, cardiac magnetic resonance; CXR, chest X-ray; ECHO, echocardiogram; MI, myocardial infarction.

The prevalence of culprit-free coronary arteries in our patient cohort $(13.0 \%)$ is similar to that described in some studies, ${ }^{4} 5$ while other studies have reported a lower rate of up to $5 \% .^{23}$ This variation can be attributed to the local arrangements for PPCI referral including the use of teletransmission in some centres and differences in patient population. Like in our hospital's protocol, where the patients are brought straight on the basis of initial diagnosis of STEMI made by the trained ambulance crew, the risk of false primary activation has to be weighed against that of missing a true STEMI. This would explain the presence of substantial number of troponin-negative patients in our cohort.

Cardiomyopathies formed the most prevalent group of alternative diagnosis in our study population $(18.1 \%)$. This was a heterogeneous group, with HCM being the most common, followed by DCM, TTCM and hypertensive heart disease. HCM is well known to be associated with myocardial ischaemia due to morphological abnormalities of the intramural coronary arterioles $^{22} 23$ and is the most common cause for sudden cardiac death in the young. ${ }^{18}$ However, presence of ischaemia in HCM is under-investigated in clinical practice and prevalence of HCM in patients presenting with suspected STEMI has been unknown.

Existence of TTCM has increasingly been recognised in patients presenting with ACS. TTCM was equally prevalent as HCM in our study cohort. Eitel et $a l^{24}$ reported 38 patients

Table 4 Different causes of death and average time to death

\begin{tabular}{lcl}
\hline Cause of death & Number, $\mathbf{n = 4 0}(\%)$ & Time to death in months $( \pm$ SD) \\
\hline Cardiac arrest & $8(20)$ & $5.5( \pm 8.5)$ \\
Heart failure & $15(37.5)$ & $10.6( \pm 13.8)$ \\
Myocardial infarction & $1(2.5)$ & $<1$ \\
Stroke & $6(15)$ & $13.4( \pm 15.6)$ \\
Pulmonary embolism & $1(2.5)$ & $<1$ \\
Cancer & $4(10)$ & $14.3( \pm 15.1)$ \\
Pulmonary infection & $3(7.5)$ & $19.0( \pm 17.9)$ \\
Renal failure & $1(2.5)$ & 9.1 \\
Unknown & $1(2.5)$ & 3.1 \\
\hline
\end{tabular}


Table 5 Predictors of all-cause mortality

\begin{tabular}{|c|c|c|c|c|}
\hline \multirow[b]{2}{*}{ Variable } & \multicolumn{2}{|l|}{ Univariable analysis } & \multicolumn{2}{|c|}{ Multivariable analysis* } \\
\hline & HR $(95 \% \mathrm{Cl})$ & $\mathrm{p}$ Value & HR $(95 \% \mathrm{Cl})$ & Value \\
\hline Aget & 1.41 (1.14 to 1.75$)$ & 0.002 & 1.39 (1.09 to 1.77$)$ & 0.007 \\
\hline Male sex & 0.82 (0.43 to 1.56$)$ & 0.54 & $1.51(0.71$ to 3.20$)$ & 0.28 \\
\hline High cholesterol & $0.78(0.36$ to 1.69$)$ & 0.53 & 0.77 (0.33 to 1.82$)$ & 0.56 \\
\hline Hypertension & $0.92(0.48$ to 1.79$)$ & 0.81 & 0.85 (0.39 to 1.85$)$ & 0.68 \\
\hline Smoking & 0.10 (0.01 to 0.72$)$ & 0.02 & 0.16 (0.02 to 1.18$)$ & 0.07 \\
\hline Diabetes & 1.44 (0.64 to 3.25$)$ & 0.38 & $1.23(0.50$ to 3.01$)$ & 0.65 \\
\hline ОНСА & 10.0 (4.19 to 23.9$)$ & $<0.001$ & 4.10 (1.41 to 11.9$)$ & 0.01 \\
\hline Raised troponin & 2.95 (1.54 to 5.66$)$ & 0.001 & 1.14 (0.51 to 2.54$)$ & 0.75 \\
\hline $\mathrm{EF}<50 \% \dagger$ & 3.79 (1.98 to 7.26$)$ & $<0.001$ & 2.97 (1.45 to 6.07$)$ & 0.003 \\
\hline
\end{tabular}

*Univariable $\mathrm{EF}$ and multivariable analysis based on 410 subjects, due to missing $\mathrm{EF}$ data.

tHR reported for a 10-year increase in age.

$E F$, ejection fraction; OHCA, out-of-hospital cardiac arrest.

with confirmed diagnosis of TTCM with CMR in 6100 patients presenting with ACS, but did not specify the number of patients with non-culprit angiogram to make a comparison. In a systemic review of previous studies, Gianni et $a l^{25}$ reported an average prevalence of $2 \%$ in both Caucasian and Japanese populations presenting with ACS.

DCM was the third most common cardiomyopathy in our study. Existence of DCM has been described in patients with ACS in other studies, ${ }^{2}{ }^{9} 26$ but in much smaller numbers. The diagnosis of DCM in this patient group may be coincidental, but would be relevant in view of adverse prognosis.

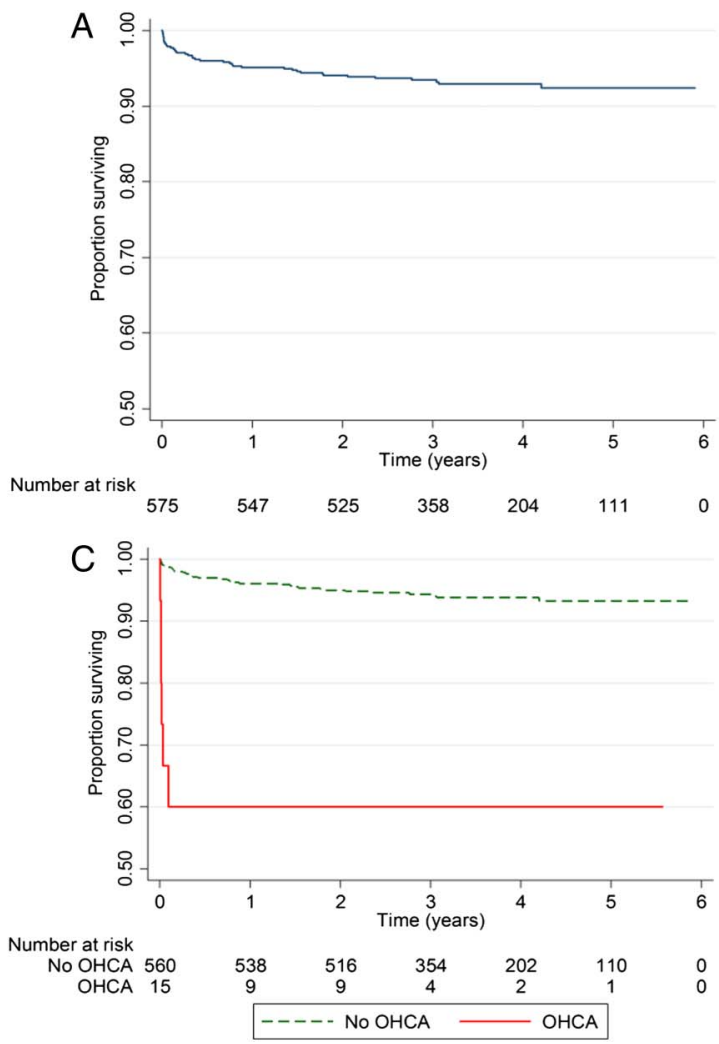

The second most common group of diagnosis was myopericarditis, but myocarditis itself was the most common condition (6.3\% in all patients and $15.2 \%$ in TP patients). Studies that have mainly focused on the usefulness of CMR in patients with normal angiograms, without reference to the total number of patients with culprit-free angiograms, usually report a much higher prevalence of myocarditis, varying between $50 \%$ and $80 \%,{ }^{8} 926$ although one study reports a prevalence of $16 \% .{ }^{10} \mathrm{~A}$ study by Larson et $a l,{ }^{4}$ which is more similar to ours demonstrated myocarditis in $19 \%$ of patients. However, the prevalence of clinical myocarditis is likely to be higher as CMR criteria have a sensitivity of up to $70 \% .^{1527}$

The diagnosis of MI in the absence of culprit disease on the angiogram has been made possible with LGE on CMR. The prevalence of MI demonstrated in studies ${ }^{4-10} 26$ comprising TP ACS patients varies from $5 \%$ to $23 \%$ (mean=13.5\%), which is similar to our patient cohort $(13.6 \%)$ if only patients who underwent CMR are included. These patients had a low mortality rate in the follow-up period (table 2).

Among the valve diseases, severe aortic valve stenosis (AVS) is well known to present with symptoms of angina. The prevalence of AVS in patients presenting as ACS for PPCI is not well known with two studies describing a single case each. ${ }^{3}{ }^{4} \mathrm{~A}$ prevalence of $2.8 \%(n=16)$ is possibly the highest reported with $75 \%$ having raised troponin.

Pulmonary abnormalities including embolism as the primary diagnosis formed the fourth largest group. Pulmonary infection was the most common followed by PE, both of which are accepted causes of raised troponin. ${ }^{6}$ The cases of lung tumours picked up on the CXR are likely to be coincidental, but would have a definite bearing on patient management and prognosis.
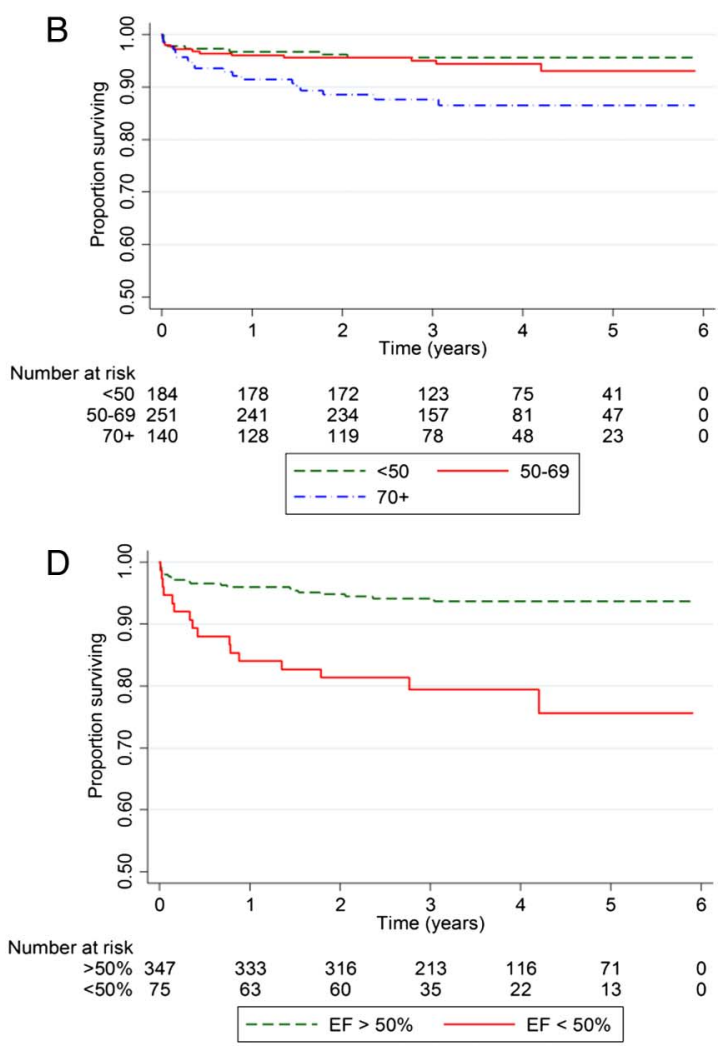

Figure 2 Kaplan-Meier survival curves (A) for the whole study group, (B) by age groups, (C) by out-of-hospital cardiac arrest (OHCA) and (D) and by ejection fraction (EF). 
Figure 3 Flow chart of suggested non-invasive imaging pathway in patients with suspected STEMI but no obstructive CAD. CAD, coronary artery disease; CXR, chest X-ray; CMR, cardiac magnetic resonance; ECHO, echocardiogram; STEMI, ST-elevation myocardial infarction.

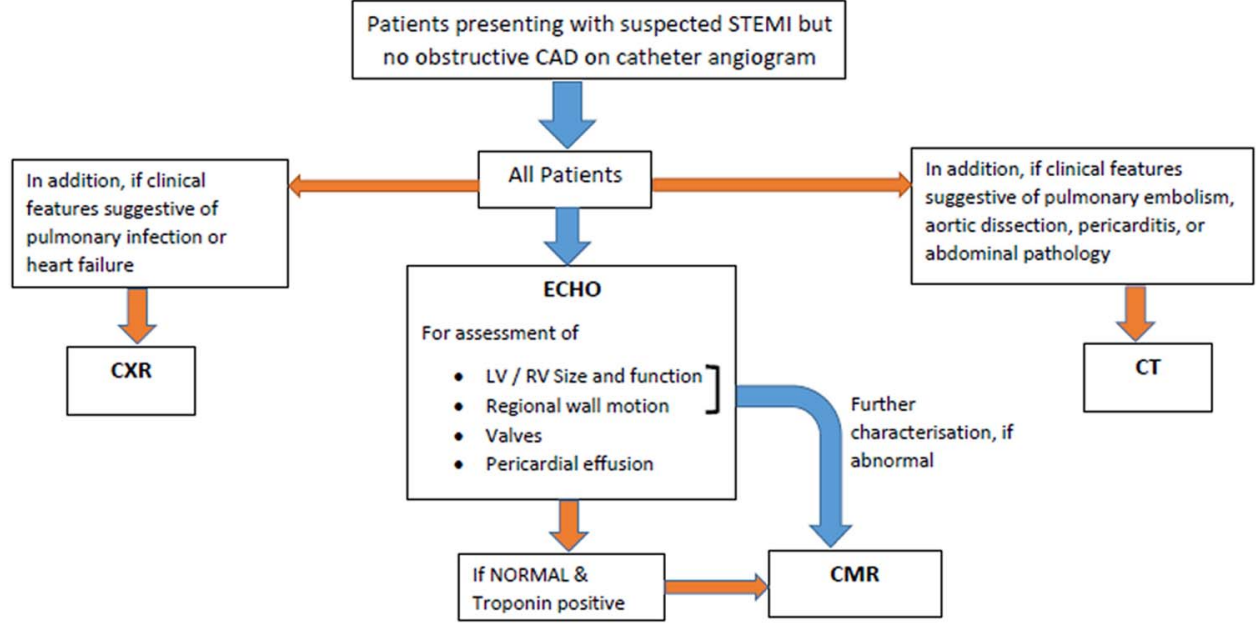

Acute aortic syndromes are well-known causes of acute chest pain with an incidence of about 6 per 100000 persons per year in a recent study. ${ }^{28}$ While the majority are identified with the appropriate clinical features and imaging, about $10 \%$ cause coronary obstruction and thus present as STEMI. ${ }^{29}$ The patients included in our study did not have coronary obstruction as patients with latter have been excluded. Lastly, a number of acute abdominal conditions including cholecystitis, pancreatitis and rupture of abdominal aortic aneurysms are known to mimic STEMI. The prevalence of these conditions is low, but early identification with imaging and management is important.

A substantial proportion of patients $(45.6 \%)$ in our study cohort did not have any other diagnosis made on clinically guided imaging, although all of them had abnormal ECGs and clinical history sufficient enough to warrant primary activation and undergo DCA. Fifty-eight of these patients also had mild rise in troponin level raising the possibility of microemboli causing myocardial necrosis, but with partial or complete recanalisation of the coronary arteries, or due to coronary embolism or spasm. ${ }^{9}$ If CMR with LGE was performed in all these patients, it is possible that some may have demonstrated areas of MI.

The study cohort contains a group of patients with heterogeneous diagnoses and hence a variable prognosis. The all-cause mortality was worse in patients with severe AVS, aortic dissection and pulmonary neoplasia, while benign in those with myocarditis. The patient with myocarditis who died was diagnosed to have giant-cell myocarditis on endocardial biopsy, which is known to have a poor outcome. However, irrespective of the diagnosis, increasing age, presentation with OHCA and EF of $<50 \%$ were associated with worse outcomes. All deaths in patients with OHCA occurred within 35 days of presentation, resulting in an excellent late survival $(60 \%)$ in those who survived (figure $2 \mathrm{C}$ ).

\section{Limitations}

The limitations of our study pertain to its retrospective observational nature. Not all patients had every type of non-invasive imaging, which would be impractical in a clinical set up. This may have led to underestimation of prevalence for certain conditions such as myocarditis and infarction; non-invasive diagnosis of which is particularly dependent upon CMR. However, we think that this underestimation would be low as the troponin levels were much lower in patients who did not undergo CMR imaging. Also even though not all patients underwent an echocardiogram, a ventriculogram was performed in every patient during DCA. This allowed identification of LV dysfunction from TTCM and other causes. Therefore, our findings are representative of a real-life scenario rather than a unified controlled approach throughout a study group. Based on our experience, we suggest a pathway for non-invasive imaging in this patient cohort (figure 3).

In conclusion, just under half of patients with suspected STEMI with a culprit-free coronary angiogram and no history of previous CAD have an alternate diagnosis made with multimodality imaging. These diagnoses are heterogeneous involving both cardiac and non-cardiac abnormalities with a variable prognosis. The use of non-invasive imaging techniques in this group of patients is, therefore, helpful as otherwise important findings could be missed and go untreated.

\section{Key messages}

What is already known on this subject?

- A proportion of patients with suspected ST-elevation myocardial infarction (STEMI) with no obstructive coronary artery disease have other diagnosis, which could be identified with one or more imaging techniques.

- There is a high prevalence of myocarditis in this patient cohort as identified on cardiac MRI studies.

What might this study add?

- About $13 \%$ of patients presenting with suspected STEMI may not have obstructive coronary artery disease. The most common alternative diagnosis in these patients as identified on multimodality imaging include cardiomyopathies (18\%), myopericarditis (8.4\%), myocardial infarction without coronary artery obstruction (4.9\%), severe valve disease (4\%), pulmonary embolism (1.2\%) and type A aortic dissection (0.7\%).

- Some of the conditions have an adverse prognosis.

How might this impact on clinical practice?

- Diagnosis of alternative diagnosis with imaging in patients with suspected STEMI can be important to implement appropriate management strategies. 
Acknowledgements The authors would like to gratefully acknowledge the statistical assistance provided by Paul Bassett and Winston Banya for this study.

Contributors TKM initiated and proposed the study. PPGR and LR collected the data. TKM, AG, SR-H and JW checked the data for their respective imaging modalities. TKM and LR performed the statistical analysis and initial draft of the manuscript. BA, TKM, LR, AG, SR-H, TK, JW and MD revised the manuscript. All authors read and approved the final manuscript.

Competing interests None declared.

Ethics approval Royal Brompton \& Harefield NHS Foundation Trust.

Provenance and peer review Not commissioned; externally peer reviewed.

Open Access This is an Open Access article distributed in accordance with the Creative Commons Attribution Non Commercial (CC BY-NC 4.0) license, which permits others to distribute, remix, adapt, build upon this work non-commercially, and license their derivative works on different terms, provided the original work is properly cited and the use is non-commercial. See: http://creativecommons.org/ licenses/by-nc/4.0/

\section{REFERENCES}

1 O'Gara PT, Kushner FG, Ascheim DD, et al. 2013 ACCF/AHA guideline for the management of ST-elevation myocardial infarction: a report of the American College of Cardiology Foundation/American Heart Association Task Force on practice guidelines. Circulation 2013;127:e362-425.

2 Widimsky P, Stellova B, Groch L, et al. Prevalence of normal coronary angiography in the acute phase of suspected ST-elevation myocardial infarction: experience from the PRAGUE studies. Can J Cardiol 2006:22:1147-52.

3 Gu YL, Svilaas T, van der Horst IC, et al. Conditions mimicking acute ST-segment elevation myocardial infarction in patients referred for primary percutaneous coronary intervention. Neth Heart J 2008;16:325-31.

4 Larson DM, Menssen KM, Sharkey SW, et al. "False-positive" cardiac catheterization laboratory activation among patients with suspected ST-segment elevation myocardial infarction. JAMA 2007;298:2754-60.

5 Prasad SB, Richards DA, Sadick N, et al. Clinical and electrocardiographic correlates of normal coronary angiography in patients referred for primary percutaneous coronary intervention. Am J Cardiol 2008;102:155-9.

6 Agewall S, Giannitsis E, Jernberg $T$, et al. Troponin elevation in coronary vs. non-coronary disease. Eur Heart J 2011;32:404-11.

7 Extramiana F. ST segment elevation: a common ECG sign for different diseases and different mechanism. J Electrocardiol 2013;46:90-1.

8 Monney PA, Sekhri N, Burchell T, et al. Acute myocarditis presenting as acute coronary syndrome: role of early cardiac magnetic resonance in its diagnosis. Heart 2011;97:1312-18.

9 Assomull RG, Lyne JC, Keenan N, et al. The role of cardiovascular magnetic resonance in patients presenting with chest pain, raised troponin, and unobstructed coronary arteries. Eur Heart J 2007;28:1242-9.

10 Mahmoudi M, Harden S, Abid N, et al. Troponin-positive chest pain with unobstructed coronary arteries: definitive differential diagnosis using cardiac MRI. Br J Radiol 2012;85:e461-6.

11 Dalby M, Kharbanda R, Ghimire G, et al. Achieving routine sub 30 minute door-to-balloon times in a high volume $24 / 7$ primary angioplasty center with autonomous ambulance diagnosis and immediate catheter laboratory access. Am Heart J 2009;158:829-35.

12 Kramer CM, Barkhausen J, Flamm SD, et al. Standardized cardiovascular magnetic resonance imaging (CMR) protocols, society for cardiovascular magnetic resonance: board of trustees task force on standardized protocols. J Cardiovasc Magn Reson 2008; 10:35.

13 Abbara S, Arbab-Zadeh A, Callister TQ, et al. SCCT guidelines for performance of coronary computed tomographic angiography: a report of the Society of Cardiovascular Computed Tomography Guidelines Committee. J Cardiovascular Comput Tomogr 2009;3:190-204.

14 Gardin JM, Adams DB, Douglas PS, et al. Recommendations for a standardized report for adult transthoracic echocardiography: a report from the American Society of Echocardiography's Nomenclature and Standards Committee and Task Force for a Standardized Echocardiography Report. J Am Soc Echocardiogr 2002;15:275-90.

15 Friedrich MG, Sechtem U, Schulz-Menger J, et al. Cardiovascular magnetic resonance in myocarditis: a JACC White Paper. J Am Coll Cardiol 2009;53:1475-87.

16 Friedrich MG. Tissue characterization of acute myocardial infarction and myocarditis by cardiac magnetic resonance. JACC Cardiovasc Imaging 2008;1:652-62.

17 Elliott $P$, Andersson $B$, Arbustini $E$, et al. Classification of the cardiomyopathies: a position statement from the European Society Of Cardiology Working Group on Myocardial and Pericardial Diseases. Eur Heart J 2008;29:270-6.

18 Gersh BJ, Maron BJ, Bonow RO, et al. 2011 ACCF/AHA guideline for the diagnosis and treatment of hypertrophic cardiomyopathy: a report of the American College of Cardiology Foundation/American Heart Association Task Force on practice guidelines. Circulation 2011;124:e783-831.

19 Bybee KA, Kara T, Prasad A, et al. Systematic review: transient left ventricular apical ballooning: a syndrome that mimics ST-segment elevation myocardial infarction. Ann Intern Med 2004;141:858-65.

20 Drazner $\mathrm{MH}$. The progression of hypertensive heart disease. Circulation 2011;123:327-34.

21 Vahanian A, Baumgartner $\mathrm{H}$, Bax J, et al. Guidelines on the management of valvular heart disease: The Task Force on the Management of Valvular Heart Disease of the European Society of Cardiology. Eur Heart J 2007;28: 230-68.

22 Maron MS, Olivotto I, Maron BJ, et al. The case for myocardial ischemia in hypertrophic cardiomyopathy. J Am Coll Cardiol 2009;54:866-75.

23 Ismail TF, Hsu LY, Greve AM, et al. Coronary microvascular ischemia in hypertrophic cardiomyopathy — a pixel-wise quantitative cardiovascular magnetic resonance perfusion study. J Cardiovasc Magn Reson 2014;16:49.

24 Eitel I, Behrendt F, Schindler K, et al. Differential diagnosis of suspected apical ballooning syndrome using contrast-enhanced magnetic resonance imaging. Eur Heart J 2008;29:2651-9.

25 Gianni M, Dentali F, Grandi AM, et al. Apical ballooning syndrome or Takotsubo cardiomyopathy: a systematic review. Eur Heart J 2006;27:1523-9.

26 Baccouche $H$, Mahrholdt $H$, Meinhardt $G$, et al. Diagnostic synergy of non-invasive cardiovascular magnetic resonance and invasive endomyocardial biopsy in troponin-positive patients without coronary artery disease. Eur Heart J 2009;30:2869-79.

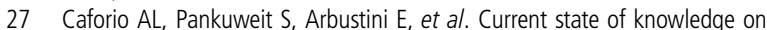
aetiology, diagnosis, management, and therapy of myocarditis: a position statement of the European Society of Cardiology Working Group on Myocardial and Pericardial Diseases. Eur Heart J 2013;34:2636-48, 48a-48d.

28 Howard DP, Banerjee A, Fairhead JF, et al. Population-based study of incidence and outcome of acute aortic dissection and premorbid risk factor control: 10-year results from the Oxford Vascular Study. Circulation 2013;127:2031-7.

29 Jánosi RA, Buck T, Erbel R. Mechanism of coronary malperfusion due to type-a aortic dissection. Herz 2009;34:478. 